\title{
Surface Reconstruction Based on Reverse Engineering and Testing
}

\author{
Shigang Wang ${ }^{\mathrm{a}}$, Yong Yan ${ }^{\mathrm{b}}$, Shufeng Jiang ${ }^{\mathrm{c}}$, Fengjuan Wang ${ }^{\mathrm{d}}$ \\ School of Mechatronics Engineering, Qiqihar University, Qiqihar, China \\ ahljwangsg@163.com, ${ }^{\mathrm{b}} 18714303670 @ 163 . c o m,{ }^{\mathrm{c} j}$ jsf349@Sina.com, ${ }^{\mathrm{d}}$ wfj320110@163.com
}

\begin{abstract}
Reverse engineering is an important part of CAD/CAM technology. Reverse engineering technology plays an important role in the highly competitive global market, the use of this technology can shorten the time from design to manufacture, which can improve the product in the international market competitiveness. This paper introduces the concept, characteristics and working process of reverse engineering, the related concepts of application of reverse engineering, reverse engineering to develop safety helmet. Through the reverse design safety helmet, expounds the point cloud data in Imageware data processing, curved surface reconstruction and modeling process. Introduced by a large number of data points for curve, surface reverse design method, and construct a model of the original point cloud contrast, error analysis and error of the reasonable results are obtained. At the same time describes the surface smoothing method of detection.
\end{abstract}

Keywords-reverse engineering; imageware; the point cloud data; surface reconstruction; helmet

\section{INTRODUCTION}

Intense competition in the global market for industrial industry put forward higher requirements, especially those of small and medium scale enterprises. These requirements include a significant reduction in product development cycle, increase product customer resistance; they need to have new technology strategies and tools to support. In order to meet the need of the development of the modern advanced manufacturing, the physical samples or manual model into CAD data, to take advantage of Rapid Prototyping system, Computer Aided manufacturing system, Product data Management and other advanced system for processing and Management, and further modification and redesign. This is when you need an integrated solution: sample $\rightarrow$ Data $\rightarrow$ samples. Reverse engineering for manufacturing provides a new efficient refactorings, implementation from real objects to the direct conversion of geometric model. Nowadays, the use of $\mathrm{CAD} / \mathrm{CAM}$ technology, advanced manufacturing techniques to real products in kind of reverse engineering has become a hot topic in CAD/CAM field and become the main content of reverse engineering applications.

\section{WORK FLOW OF REVERSE ENGINEERING}

Reverse engineering can be called reverse engineering, reverse engineering, also known measure of manufacturing, in a sense, reverse engineering is a specific way to complete the work of a physical model of imitation. It is digital technology that are relevant to physical objects into a CAD model, geometric model reconstruction of the floorboard of the technology and products manufacturing technology, is to convert existing products or mock-up to engineering design model and concept model, and on this basis to anatomy, deepen the existing products, and the creative process. It originated in the precision measurement and quality inspection, is the design of downstream feedback to upstream circuit design.

First, the general process of reverse engineering is to obtain product information by measuring the physical scanning and a variety of advanced data processing means for converting CAD models for physical prototypes, and then use computer-aided manufacturing, rapid prototyping manufacturing, rapid tooling and PDM systems and other advanced technologies be a system procedure process.

\section{DATA ACQUISITION}

In the surface of digital technology, according to the different test methods, data acquisition method can be divided into two categories: contact measurement and noncontact measurement. Contact measurements are generally measured using a variety of probe data. On-contact measurement is mainly based on the basic principle in the field of optical, acoustic, magnetism and so on, to a certain amount of physical simulation through the proper algorithm is converted into the coordinate points on the surface of the sample.

Here we use a non-contact three scanners for data acquisition. It combines optical triangulation, fringe projection; phase shift and other principles for physical prototypes do non-contact measurement. The measurement speed, high-resolution scanning, data point's intensive, high precision, suitable for measuring the external surfaces of complex parts of the model. Fig. 1 is the point cloud data on the safety cap scanning.

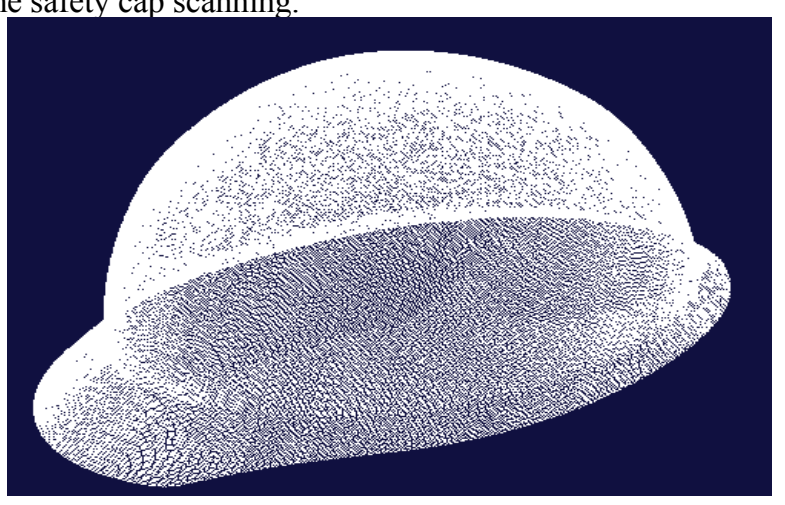

Figure 1. Helmet point cloud data 


\section{SURFACE RECONSTRUCTION}

Surface reconstruction is the use of scattered point data on the surface of the product, or by interpolation fitting an approximate model is constructed to approximate product prototypes. According to different topology form, the current research in reverse engineering, free-form surface modeling methods are divided into triangular Bezier surface based surface construct method and NURBS (non-uniform rational b-spline) surface based on rectangular domain parameters of the curved surface fitting method. On the basis of data segmentation, first identify different kinds of point cloud data, and then according to different types of point cloud model, choose a different method of building surface. Imageware in surface structure is finished based on NURBS, its mathematical model is:

$$
p(u, v)=\frac{\sum_{i=0}^{m} \sum_{j=0}^{n} w_{i, j} d_{i, j} N_{i, k}(u) N_{j, l}(v)}{\sum_{i=0}^{m} \sum_{j=0}^{n} w_{i, j} N_{i, k}(u) N_{j, l}(v)}
$$

In the formula:

$d_{i j}$-Control vertices, a topological rectangular array to form a control grid, $i=0,1 \cdots m, j=0,1 \cdots n ; w_{i j}$-Power factor associated with vertex $d_{i j}$, four vertices with positive power factor, the $w_{0,0}, w_{n, 0}, w_{0, n}, w_{n, n}>0$, the rest $w_{i, j} \geq 0$, and order right of $k l$ factor cannot be zero at the same time; $N_{i, k}(u), N_{j, l}(v)$-are $u$ to $k$ and $v$ to $l$ times of the specification of the B-spline.

\section{IMAGEWARE OF POINT CLOUD DATA PROCESSING}

Imageware is currently the most widely used in the reverse engineering software, with a strong point processing features, with a strong measurement data processing, surface modeling, and the function of error detection, can handle tens of thousands to millions of point cloud data. Being widely used in the automotive, aviation, aerospace, consumer appliances, mold, computer parts, such as design and manufacturing. Here we use the imageware fitting the above point cloud data of helmet.

\section{POINT CLOUD BLOCK}

In order to make the reverse design of more accurate and simple, we usually want to block complex point cloud data. Point cloud data block reasonable, not only can save time by using the reverse design, and can reduce the error point cloud and fitting between surfaces. According to the characteristics of the helmet of the point cloud by means of curvature analysis, we can according to the different curvature, the point cloud data of safety helmet can be divided into two point cloud, the cap body, brim two parts (as shown in Fig. 2). Gap between the body and the cap brim point cloud, in the fitting surface, we will be generated by rounding function.

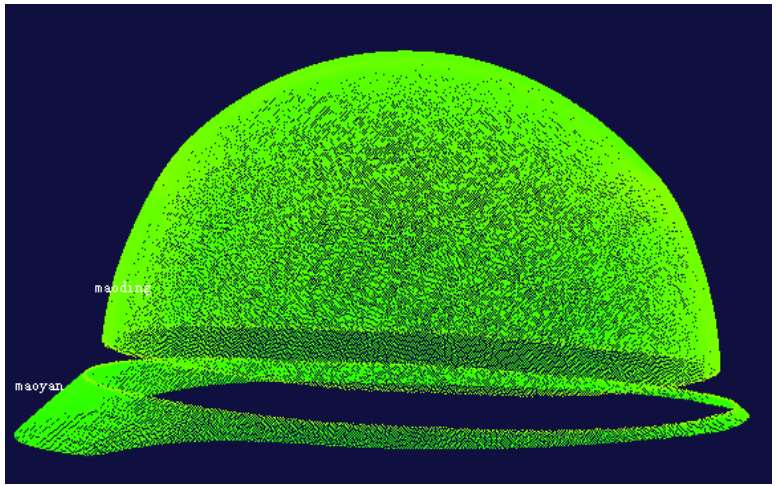

Figure 2. Point cloud data segmentation

\section{FITTING SURFACES}

Imageware software mainly adopts dot - surface and point - line - face, two methods of quadric surface, transition surface and free surface fitting.

\section{A. Point-surface Fitting Method}

Of point Cloud data collected when the conditions for the Uniform Surface or when reaching into a point Cloud data plane, cylinder, sphere, cone and other geometric shapes, you can use "the Construct - Surface from the Cloud - Uniform sur - face" instructions to generate a Uniform Surface directly. Point cloud peak part is in this way direct fitting synthetic surface (Fig. 3).

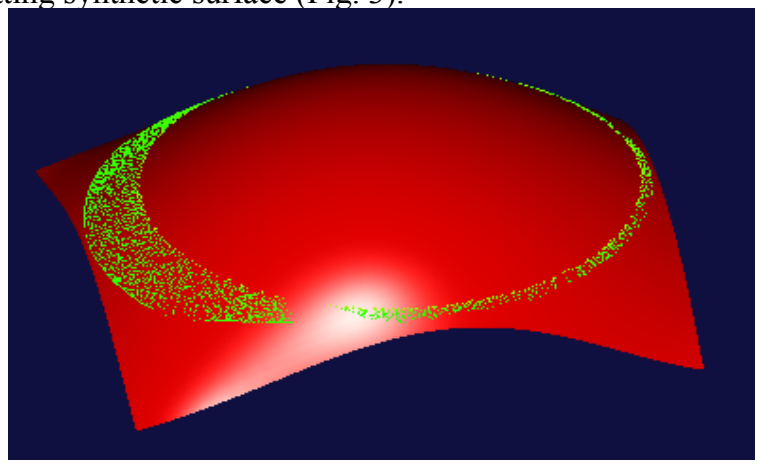

Figure 3. Hat complete point cloud and surface fitting

\section{B. Point-line-plane Fitting Method}

This method is often used when finished surface reconstruction of a method. Not all of the distribution of point cloud data is smooth, sometimes change of curvature is bigger, the point cloud data directly to composite surface will appear large deviation, and we can not get the desired surface. In this case, we can first point cloud based on the contour feature point cloud data fitting out, and then fitted to the surface via contour. In this case, according to the features of point cloud cap body, we will helmet extract contour line from point cloud (Fig. 4), and then according to the contour in the cap body surface fitting and brim of pruning. Fig. 5 has not been trimmed surfaces after complete fitting. 


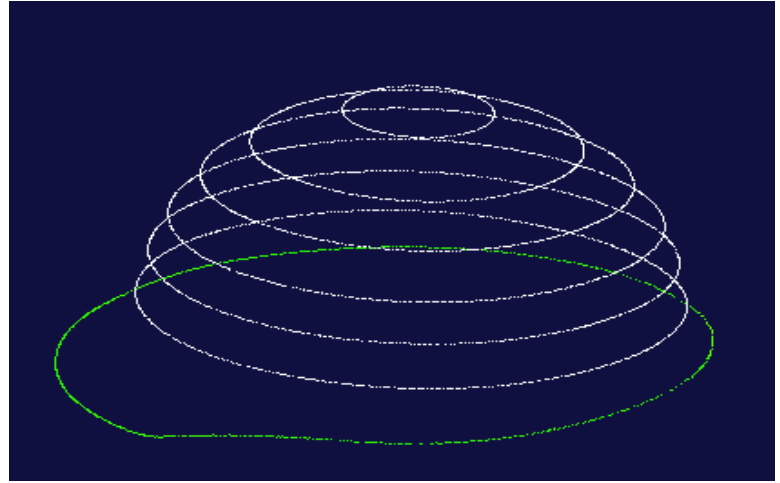

Figure 4. Helmet contour line

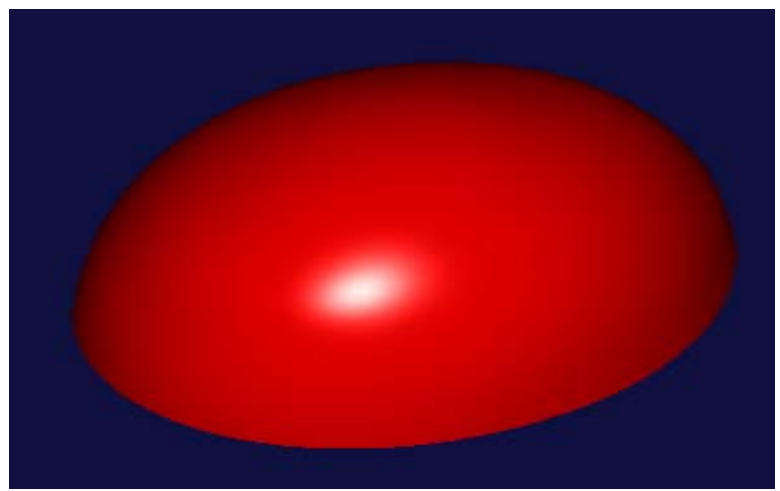

Figure 5. Fitting complete surface

\section{TRIMMED SURFACES}

After completion of surface fitting, to cut off the excess surface boundary, or for chamfering two surfaces. Surface helmet brim portion can be trimmed based on the contour curve of the brim, and then the curved portion of the cap body extends, again with the brim portion is chamfered surfaces. Finally finished fitting helmet is shown in Fig. 6.

\section{ERROR DETECTION}

After curve fitting is completed, we want to test the error between the Surface and the point Cloud, in imageware, error is detected by "Measure - Surface to - Cloud difference" instructions to complete (as shown in Fig. 7). If the fitting error between the surface and the point cloud is large, we have to re-surface fitting or fine-tuning, to reduce the error to achieve the required accuracy.

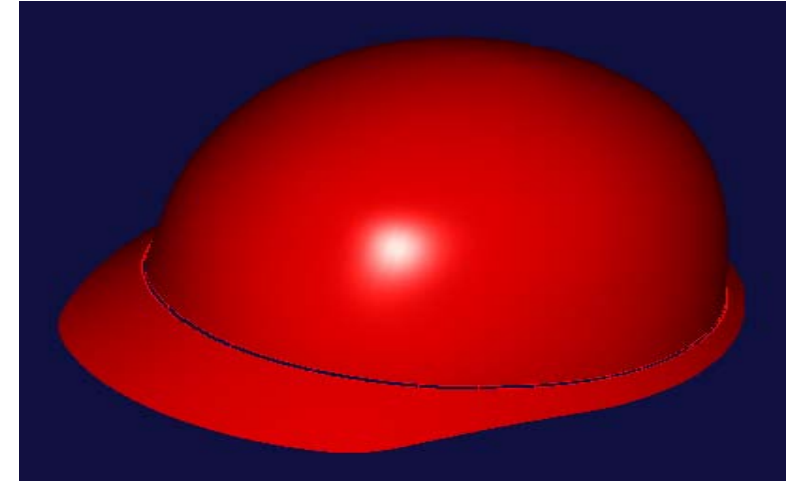

Figure 6. Completely fitting helmet

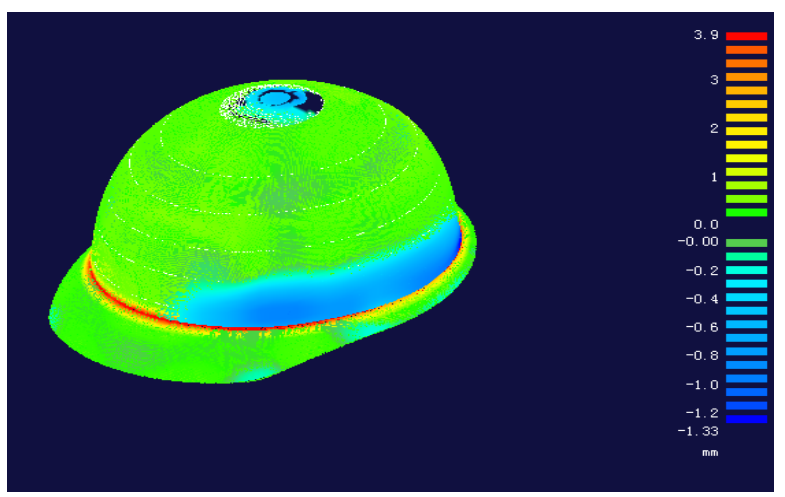

Figure 7. Error detection

The degree of smoothing and curvature continuity check

\section{A. Curvature Comb Method}

Inspection of surface curvature comb can determine surface curvature continuity, curvature comb length in the direction of curvature between adjacent needles and reflect the direction of surface curvature and the curvature values. In general, the curvature of the needle on a comb with curvature toward the same direction, and the length of the ups and downs of the curvature of the needle is unlikely that such surface fairing better. In imageware, we can use the "Evaluate-Curvature-Surface Needle" command can test $\mathrm{x}, \mathrm{y}$, $\mathrm{z}$ three directions of the curvature comb (Figure 8). Curvature of the needle length of the curved portion of the cap body basically the same, so the cap body surface smoothness is good.

\section{B. Reflection Contour Detection Method}

After the contour to appear on the surface, rotate to view every Angle contour, contour of the intersection of generally does not happen, smoothing of surface is achieved. Using the command "Evaluate-Surface Flow-Reflection Lines" by contour method to detect surface smoothness (Fig. 9). 


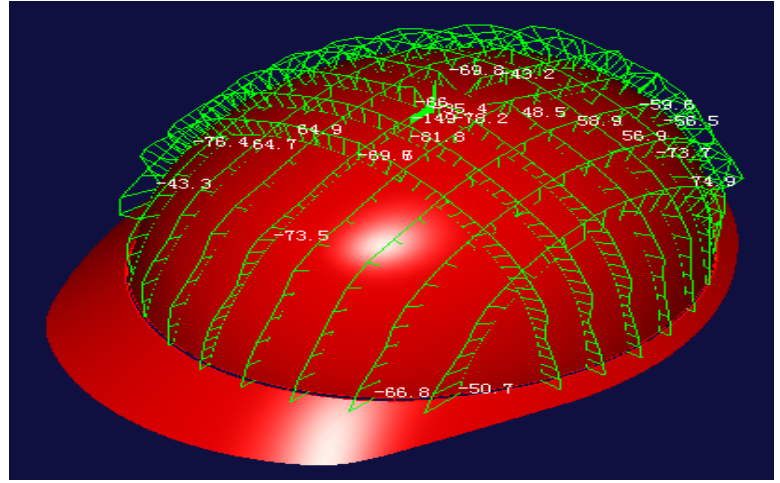

Figure 8. Curvature comb detection

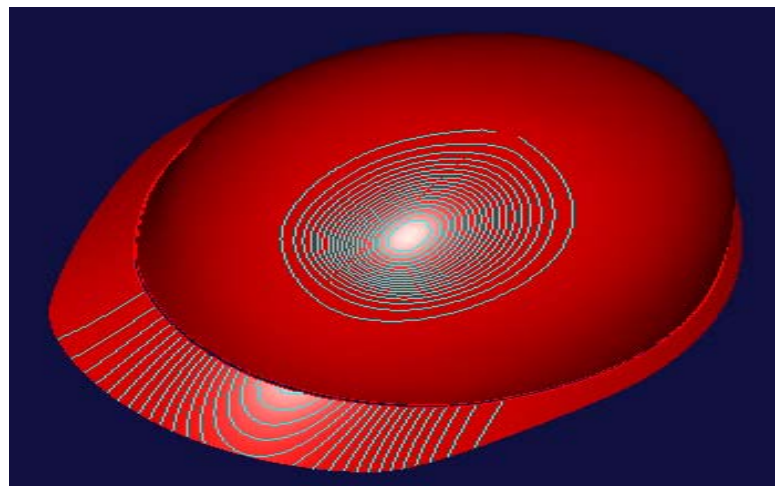

Figure 9. Contour detection

\section{CONCLUSIONS}

By reverse engineering instance helmets, reverse engineering technology and existing CAD/CAM system, the relationship between applications and reverse engineering of a brief introduction, but also for enterprises reverse engineering product design and mold manufacturing provides a good idea. Imageware software in reverse design of powerful almost can finish the point cloud processing, data partition, curve and curved surface reconstruction and curved surface quality detection, the whole process of testing the surface quality of reverse design. Therefore, imageware in reverse design will have a very broad application prospects.

\section{ACKNOWLEDGMENT}

This work was supported by Project of Department of Education of Heilongjiang Province, China (Grant No. 12521597) and Qiqihar University youth teacher research startup project (Grant No. 2014k-M04).

\section{REFERENCES}

[1] Yuanqing Jiang, Peijun Liu, "UG/Imageware reverse engineering training course," Beijing: Tsinghua University Press, 2003.

[2] Yong Li, Jinjiang Wu, Yuanyuan Xie, "ImageWare based reverse engineering techniques," Mechanical Engineering and Automation, No. 3, 2008, pp. 78-80.
[3] Yingchun $\mathrm{Wu}$, Guoliang Zhang, "Modeling based on reverse engineering of artificial knee joint," Science Technology and Engineering, vol. 10, 2010, pp. 3883-3887.

[4] Zhongzhen Long, Pizhu Yu, "Technology is based on reverse engineering in the design of car headlights," No. 4, 2010, pp. 43-47.

[5] Caixia Wang, "Based on reverse engineering data analysis," Xi'an Aviation Technical College, vol. 28, 2010, pp. 15-16.

[6] Wei Jiang, "Application of reverse engineering technology in the manufacture of mechanical parts," Technology Consulting (Decision Management), No. 4, 2010, pp. 72.

[7] Dan Jiang, Lancheng Wang, “An algorithm of NURBS surface fitting for reverse engineering," Int J Adv Manuf Technol, vol. 31, 2006, pp. 92-97. 